\title{
Author Correction: How we created neuromorphic engineering
}

Carver Mead

Correction to: Nature Electronics https://doi.org/10.1038/s41928-020-0448-2, published online 21 July 2020.

In the version of this Reverse Engineering article originally published, in the sentence beginning "So how is it that the common housefly, with a few hundred neurons", the number of neurons was incorrect, and should have read "a hundred thousand neurons". The article has now been updated.

Published online: 17 August 2020

https://doi.org/10.1038/s41928-020-00471-y

๑) Springer Nature Limited 2020 